\title{
World Journal of Kidney structure and function in
Pediatric Surgery dilating vesicoureteral reflux patients with anorectal malformation
}

Yuichiro Miyake, Hiroyuki Koga, Geoffrey J Lane, Atsuyuki Yamataka

To cite: Miyake Y, Koga $\mathrm{H}$, Lane GJ, et al. Kidney structure and function in dilating vesicoureteral reflux patients with anorectal malformation. World $\mathrm{Jn}$ Ped Surgery 2020;3:e000088. doi:10.1136/wjps-2019-000088

Received 10 September 2019 Revised 15 June 2020

Accepted 15 June 2020
Check for updates

\section{(c) Author(s) (or their} employer(s)) 2020. Re-use permitted under CC BY-NC. No commercial re-use. See rights and permissions. Published by BMJ.

Department of Pediatirc General and Urogenital Surgery, Juntendo University School of Medicine, Bunkyo-ku, Tokyo, Japan

Correspondence to Dr Hiroyuki Koga; h-koga@ juntendo.ac.jp

\section{ABSTRACT}

Background We assessed the impact of anorectal malformation (ARM) on the kidneys of children with dilating vesicoureteral reflux (D-VUR) $\geq$ grade III using a simple dimercaptosuccinic acid (DMSA) scintigraphy scan based renal dysfunction score (RDS).

Methods The medical records of 121 patients with D-VUR treated between 2000 and 2014 were reviewed retrospectively. After excluding patients with secondary D-VUR $(n=18)$, presence of ARM was used to create two groups: $A R M+(n=12$ cases; 15 ureters) and ARM- $(n=91$ cases; 131 ureters). Types of ARM, grades of D-VUR, bladder and bowel dysfunction (BBD) and RDS were compared.

Results Patient demographics, mean follow-up, grades of D-VUR and history of urinary tract infections were not significantly different. BBD was significantly higher in $A R M+(41.7 \%$ versus $7.7 \%, p=0.0006)$. RDS was significantly higher in ARM+ $(p=0.036)$. Grades of $D-V U R$ were significantly lower in ARM- with low RDS ( $p=0.008)$. During follow-up, changes in DMSA uptake over time were not observed in ARM+.

Conclusions While renal cortical lesions were correlated with grade of D-VUR in ARM- and RDS was significantly higher in ARM+, BBD did not appear to contribute to progressive renal dysfunction as is commonly believed. In fact, no progression in renal cortical lesions was observed in ARM + based on RDS data. Renal cortical lesions may possibly be a feature of ARM, a topic that warrants further study.

\section{INTRODUCTION}

The incidence of vesicoureteral reflux (VUR), a common anomaly of the urinary tract in childhood, is higher in patients with anorectal malformation (ARM) ${ }^{1-3}$ There is an increased risk for VUR with 'high' ARM anomalies, ranging from $33 \%$ to $54 \%$, compared with 'low' ARM anomalies, ranging from $20 \%$ to $37 \% .^{3-5}$ Because the incidence of urinary tract infections (UTIs) increases with the grade of VUR, contributing to the acquisition of renal cortical lesions that are ultimately detrimental to stable renal function over time, ${ }^{4}$ we are curious to assess the connection between bladder and bowel dysfunction (BBD; defined in detail in the
Key messages

What is already known about this subject?

- The incidence of vesicoureteral reflux (VUR) is higher in anorectal malformation (ARM) patients.

- VUR certainly increases the risk of developing symptomatic urinary tract infection (UTI), and dilating VUR is detrimental to kidney function by inference.

- Bladder and bowel dysfunction (BBD) is known to increase the risk for UTI in children.

\section{What are the new findings?}

- Patients with ARM had significantly worse renal dysfunction than patients without ARM despite both groups having similar grades of VUR and incidences of UTI.

- The incidence of BBD in patients with ARM was significantly higher than that in patients without ARM.

- The distribution of renal cortical lesions on dimercaptosuccinic acid scans did not appear to change over time in patients with ARM.

How might it impact on clinical practice in the foreseeable future?

- Follow-up should focus on meticulous assessment to identify circumstances that may stress renal function and exacerbate cortical lesions, prevent UTI and commence treatment/intervention for any condition as soon as possible.

Methods section) as a possible additional factor in the etiology of UTI in children with ARM. ${ }^{4-6}$ By understanding the relationships between VUR, UTI, ARM and renal cortical lesions, health professionals may be better prepared to predict prognosis and enhance the follow-up of VUR patients with ARM.

To the best of our knowledge, there are no comparative studies in the English literature that assess the etiologic links between BBD, VUR, UTI and ARM.

\section{METHODS}

We retrospectively reviewed the medical records of 121 consecutive VUR > grade III (dilating VUR or D-VUR) patients treated at our institute between 2000 and 2014. Eighteen 


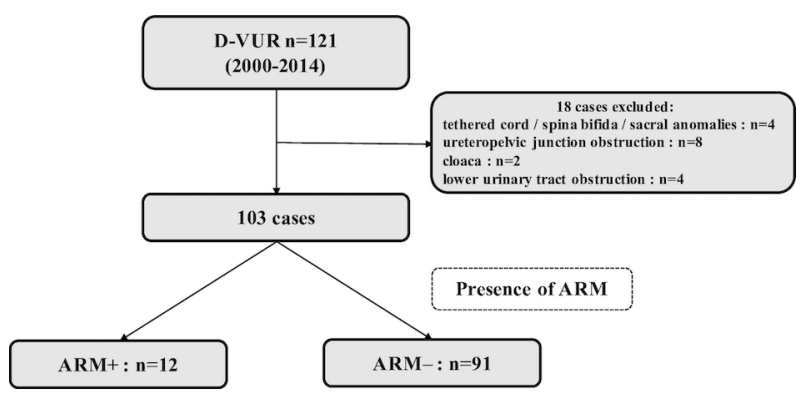

Figure 1 Subject flow chart. ARM, anorectal malformation; D-VUR, dilating vesicoureteral reflux.

patients with neurogenic bladder due to tethered cord, spina bifida and sacral anomalies, ureteropelvic junction obstruction, cloaca or lower urinary tract obstruction were excluded as being secondary causes of VUR, leaving 103 patients as subjects for this study. Subjects were divided into two groups according to the presence of ARM (figure 1) to give ARM+ $(\mathrm{n}=12 ; 15$ ureters) and ARM- ( $\mathrm{n}=91 ; 131$ ureters $)$.

Data for gender, type of ARM, grade of D-VUR assessed by using voiding cystourethrogram (VCUG), incidence of UTI (defined as fever $>38^{\circ} \mathrm{C}$ accompanied by leukocytosis with left shift, and positive catheterized urine culture growing at least $10^{5}$ bacteria per $\mathrm{mL}$ ), use of prophylactic antibiotics to prevent UTI, presence of BBD (defined by the American Urological Association as a combination of at least one lower urinary tract symptom, such as presence of urinary frequency, urinary urgency, extended periods between voiding, daytime wetting, perineal or penile pain or holding maneuvers, with at least one type of dysfunctional defecation, such as constipation, or encopresis) reported by the parents or the patient in lay terms, without specific use of classification tools, duration of follow-up and a simple renal dysfunction score (RDS), based on a format approved by the Japanese Reflux Nephropathy Forum involving scoring renal cortical lesions detected on dimercaptosuccinic acid (DMSA) scintigraphy on a scale of $0-2$ with 0 representing no cortical lesions, 1 representing less than two lesions or split renal function $>40 \%$ and 2 representing split renal function $<40 \%$ with a subclassification of $2 \mathrm{~m}$ for multiple lesions and $2 \mathrm{~d}$ for diffuse lesions were compared. ${ }^{7} \mathrm{~A}$ DMSA scan showing poor renal function with severe multiple and diffuse renal cortical lesions would thus be given an RDS of $2 \mathrm{~m}, 2 \mathrm{~d}$. Note there was no provision in the RDS used in this study to determine if renal cortical lesions were congenital or acquired (table 1).

As part of our routine protocol for evaluating patients with ARM preoperatively for definitive corrective surgery, we performed a VCUG and contrast enema to diagnose and classify the ARM. VUR was usually diagnosed at this time, and a baseline DMSA scan was performed. By the time of preoperative assessment for ARM, almost all patients had experienced UTI as a consequence of their VUR (ARM+: 10/12 cases, ARM-: 89/91 cases). While prenatally diagnosed ARM was an indication for more intensive management during pregnancy, prenatally diagnosed hydronephrosis did not tend to warrant any extra attention unless it was severe, so only a few ARMwere diagnosed with hydronephrosis prenatally, and in most ARM-, VCUG and DMSA were performed only

\begin{tabular}{|c|c|c|c|}
\hline Variables & $A R M+(n=12)$ & ARM- $(n=91)$ & $P$ value \\
\hline Male:female ratio (\% male) & $6: 6(50)$ & $57: 34(63)$ & 0.399 \\
\hline Mean incidence of all UTI per patient (including preoperative)* & $2.0 \pm 1.6$ & $2.0 \pm 1.2$ & 0.775 \\
\hline Use of prophylactic antibiotics, $n(\%)$ & $9(75)$ & $84(92)$ & 0.091 \\
\hline Recurrence of UTI, n(\%) & $8(67)$ & $61(67)$ & 0.979 \\
\hline Bilateral VUR, $\mathrm{n}(\%)$ & $3(25)$ & $36(40)$ & 0.528 \\
\hline Severity of $D-V U R, n=$ ureters $(\%)$ & & & 0.174 \\
\hline Grade III & $n=9(60)$ & $n=56(43)$ & \\
\hline Grade IV & $\mathrm{n}=3(20)$ & $\mathrm{n}=59(45)$ & \\
\hline Grade V & $n=3(20)$ & $n=16(12)$ & \\
\hline RDS n=kidneys (\%) & & & 0.036 \\
\hline RDS=0 (none) & $\mathrm{n}=2(13.3)$ & $\mathrm{n}=47(35.9)$ & \\
\hline RDS $=1$ (less than two lesions, split renal function $>40 \%$ ) & $\mathrm{n}=4(26.7)$ & $\mathrm{n}=52(39.7)$ & \\
\hline $\mathrm{RDS}=2 \mathrm{~m}$ (multiple lesions, split renal function $<40 \%$ ) & $\mathrm{n}=2(13.3)$ & $\mathrm{n}=7(5.3)$ & \\
\hline RDS=2d (diffuse lesions, split renal function <40\%) & $\mathrm{n}=7(46.7)$ & $\mathrm{n}=25(19.1)$ & \\
\hline Presence of BBD, $n(\%)$ & $5(42)$ & $7(8)$ & 0.0006 \\
\hline Mean follow-up period (years)* & $6.6 \pm 1.1$ & $6.0 \pm 0.3$ & 0.950 \\
\hline
\end{tabular}

* Data were presented with mean \pm SD.

ARM, anorectal malformation; BBD, bladder and bowel dysfunction; D-VUR, dilating vesicoureteral reflux; RDS, renal dysfunction score; SD, standard deviation; UTI, urinary tract infection; VUR, vesicoureteral reflux. 
after the first UTI, with the result that most ARM- cases did not routinely have baseline DMSA studies. Thus, while ARM+ had DMSA scans performed before and after definitive corrective surgery, ARM- did not. Similarly, during postoperative follow-up, DMSA scans were performed at least 3 months after the last UTI in order to avoid documenting acute renal cortical lesions and at least every 2 years in all patients to update the status of renal cortical lesions.

Data were expressed as mean \pm standard deviation (SD) or median value. The Mann-Whitney $\mathrm{U}$ test, the unpaired $t$-test, the $\chi^{2}$ test and the one-way analysis of variance test were used for statistical analysis. A p value less than 0.05 was considered to be statistically significant.

\section{RESULTS}

All ARM cases were postoperative for definitive corrective surgery at the time data were collected. All ARM+ patients were fully toilet trained at the time of BBD assessment. All urine samples were obtained by urinary catheterization.

Types of ARM in ARM+ $(n=12)$ were 'high' $(n=1$; two ureters), 'intermediate' $(n=7$; eight ureters $)$ and 'low' ( $\mathrm{n}=4$; five ureters). Differences between ARM+ and ARM- for sex distribution (male: $50 \%$ vs $62.6 \%$ ), mean incidence of all UTI (including preoperative) per patient (2.00 vs 2.04), incidence of bilateral D-VUR (25\% vs $44.0 \%)$ or mean overall duration of follow-up [defined as the period from the initial consultation to the final consultation at our institute; $6.6 \pm 1.1$ years (range: 2.2-14.6) vs $6.0 \pm 0.3$ years (range: $1.6-10.7$ ) ] and grades of D-VUR were not significant (table 1). The incidence of $\mathrm{BBD}$ in $\mathrm{ARM}+$ was significantly higher than that in ARM- $(41.7 \%$ vs $7.8 \%$; $\mathrm{p}=0.0006)$ (table 1$)$. However, differences in the mean ages of BBD patients with respect to ARM were not significantly different $[6.9 \pm 2.6$ years (range: $4.7-11.8$ ) for ARM+ versus $7.2 \pm 1.9$ years (range: 4.0-9.5) for ARM-, $\mathrm{p}=0.53$ ]. DMSA scans indicated that both severe multiple and diffuse renal cortical lesions (RDS: 2m, 2d) were identified significantly more often in ARM+ than in ARM- $(60.0 \%$ versus $24.4 \%, \mathrm{p}=0.036)$ (table 1). We also observed that there was no tendency

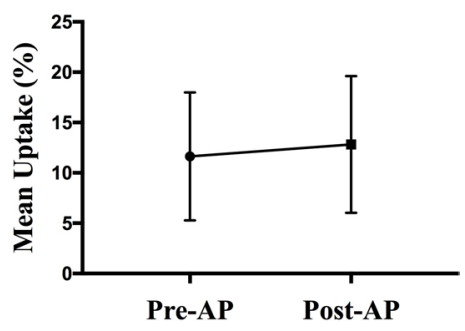

Figure 2 Renal function in postoperative ARM+. Mean uptake on DMSA scintigraphy did not deteriorate after definitive corrective surgery for ARM+. ARM, anorectal malformation; pre-AP, preanorectoplasty; post-AP, postanorectoplasty. $n=10$ kidneys. for mean uptake on DMSA scintigraphy to deteriorate after definitive corrective surgery in ARM+ (figure 2).

Duration of follow-up was hard to standardize because we did not have a protocol for follow-up. We calculated the mean duration of follow-up of post-ARM surgery patients with complete DMSA results, but the figure, 4.2 years (range: 1.0 to 14.9 years), was not specific enough to use meaningfully, so we abandoned using it as a criterion for comparison.

To summarize, differences in sex distribution, mean incidence of all UTI per patient, mean age of patients, grades of D-VUR, incidence of BBD in ARM+ according to RDS and RDS for different types of ARM were not significant. Although the overall spectrum of grades of D-VUR was not significantly different, grade of D-VUR was significantly lower in ARM- with low RDS ( $p=0.0079)$. In other words, there were less renal cortical lesions in more normal patients (see table 2 for RDS results according to sex, age and grade of VUR).

\section{DISCUSSION}

To the best of our knowledge, this is the first study to investigate correlations between D-VUR, BBD, RDS and ARM. In this study, DMSA identified diffuse renal cortical lesions in the kidneys of as many as $46.7 \%$ of ARM+. ARM+ also had significantly higher RDS than ARM- when differences in grades of D-VUR and incidences of UTI were not significantly different, and there were no differences in RDS related to type of ARM. In other words, RDS results were a consequence of the presence of ARM, not the type of ARM. Most strikingly, the distribution of renal cortical lesions on DMSA scans as reflected by RDS did not appear to change over time in $\mathrm{ARM}+$, which was contrary to expectations. The fact that renal cortical lesions did not progress during follow-up suggested that renal cortical lesions were most likely to be stable as scars.

Renal cortical lesions are known to be associated with D-VUR secondary to UTI because of anatomic anomalies associated with VUR, ${ }^{8}$ or because of bladder dysfunction that has been reported in D-VUR as part of a congenital VUR anomaly. ${ }^{9}$ VUR certainly increases the risk of developing symptomatic UTI, ${ }^{4}$ so the presence of D-VUR should be detrimental to kidney function. Capozza $e t a l^{10}$ documented that there was a different natural history for boys and girls with VUR. Sillén ${ }^{11}$ studied VUR in infancy and found that grade V VUR was almost exclusively male and that the resolution rate for grade IV VUR was $40 \%-50 \%$ during the first year of life. Wennerström et $a l^{12}$ studied the incidence of UTI sequelae and found that boys tended to have reflux-associated renal cortical lesions, while girls tended to have UTI-related cortical lesions. Although we did not observe gender differences in this study, we did observe that renal cortical lesions were correlated with grade of VUR in ARM-, with RDS=0 being predominant when D-VUR grades were lower $(\mathrm{p}=0.008)($ table 2$)$. 


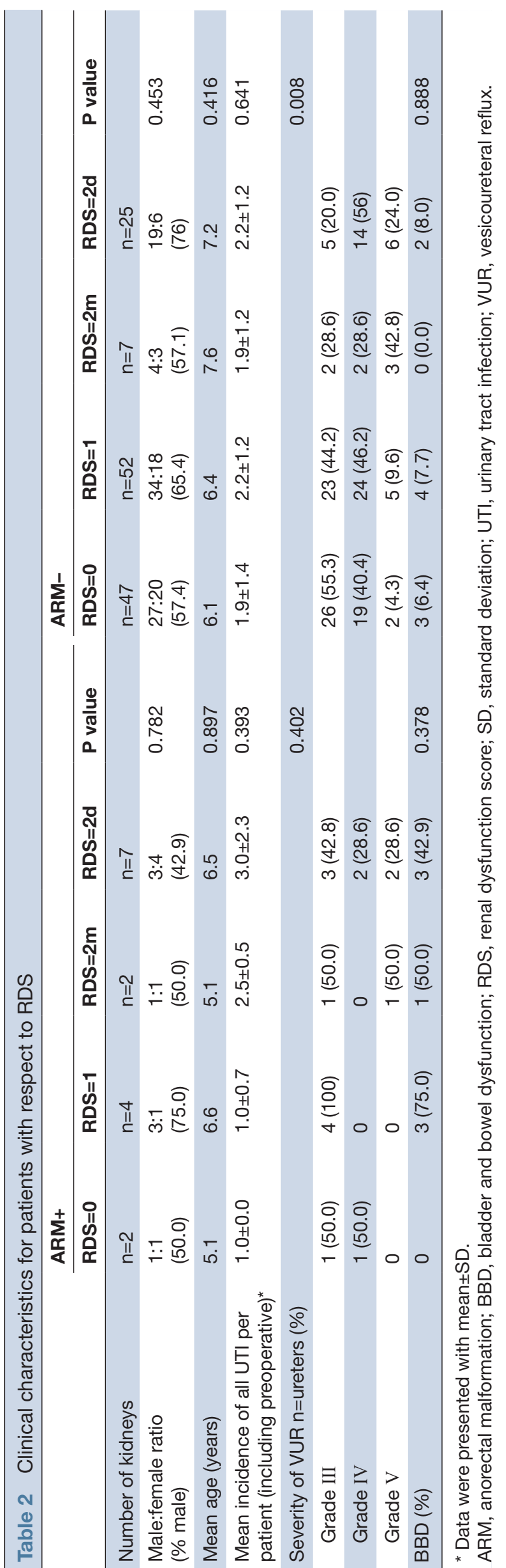

Increased awareness of the association between BBD and D-VUR led the American Urological Association to report that BBD increased the risk for breakthrough UTI in children receiving antibiotic prophylaxis, reduced the success rate for endoscopic injection therapy, increased the risk for postoperative UTI and was a risk factor for renal scarring. ${ }^{45}$ In this series, as many as $41.7 \%$ of our fully toilet trained ARM+ patients had BBD that started initially with severe constipation requiring enemas or irrigations after anorectoplasty. In addition, constipation was reported in postoperative ARM patients, ranging from $38 \%$ to $46 \%$ purportedly related to persistent anatomic anomalies of the anorectum. ${ }^{13} 14$ Thus, BBD and postoperative constipation might influence kidney structure and function, and we were careful to follow-up our ARM+ with BBD patients regularly for evaluation of BBD and renal/urinary tract function, and if necessary, prescribed appropriate treatment to minimize the symptoms of $\mathrm{BBD}$. However, there was also no change in the distribution of cortical lesions on DMSA during follow-up based on the presence of $\mathrm{BBD}$, which again suggested that renal cortical lesions were stable and not progressive.

Unfortunately, our study was limited because it was retrospective and performed at a single institute. We also did not have baseline data of DSMA lesions without a history of UTI, and our sample size was too small for us to make any categorical conclusion, ${ }^{715}$ but our data were sufficient to identify trends in various criteria (demographic data and history of UTI) that were not statistically different.

By assessing the known risk factors for renal cortical lesions in D-VUR patients with respect to ARM, we hypothesize that the renal cortical lesions seen in ARM patients may be an inherent feature of the anorectal anomalies comprising ARM itself, rather than being acquired or secondary. In other words, our study demonstrates that diffuse renal cortical lesions may, in fact, be part of the clinical spectrum that manifests clinically as ARM.

To validate our results, further data on the distribution of lesions need to be collected by performing DMSA routinely when ARM is newly diagnosed, followed by regular studies over time. By doing this, if lesions are present on initial DMSA studies, they can be classified as being congenital, but should no lesions be identified, then lesions must be classified as being acquired, and if they persist on repeat DMSA performed at least 3 months following an episode of acute UTI, then they should be considered to be scars. Even though there was no progression of renal cortical lesions observed during this study, follow-up should be regular, and treatment/ intervention should commence as early as possible when required.

Contributors MY collected and analyzed date and drafted the manuscript. KH served as scientific advisers. LGJ reviewed the English and study design. YA critically reviewed the study proposal.

Funding The authors have not declared a specific grant for this research from any funding agency in the public, commercial or not-for-profit sectors. 
Competing interests None declared.

Patient consent for publication Not required.

Ethics approval This study was approved by the Juntendo University School of Medicine Institutional Review Board and complies with the Helsinki Declaration of 1975 (revised 1983).

Provenance and peer review Not commissioned; externally peer reviewed.

Data availability statement Data are available on reasonable request.

Open access This is an open access article distributed in accordance with the Creative Commons Attribution Non Commercial (CC BY-NC 4.0) license, which permits others to distribute, remix, adapt, build upon this work non-commercially, and license their derivative works on different terms, provided the original work is properly cited, appropriate credit is given, any changes made indicated, and the use is non-commercial. See: http://creativecommons.org/licenses/by-nc/4.0/.

\section{REFERENCES}

1 Sanchez S, Ricca R, Joyner B, et al. Vesicoureteral reflux and febrile urinary tract infections in anorectal malformations: a retrospective review. J Pediatr Surg 2014;49:91-4.

2 Ganesan I, Rajah S. Urological anomalies and chronic kidney disease in children with anorectal malformations. Pediatr Nephrol 2012;27:1125-30.

3 Goossens WJH, de Blaauw I, Wijnen MH, et al. Urological anomalies in anorectal malformations in the Netherlands: effects of screening all patients on long-term outcome. Pediatr Surg Int 2011;27:1091-7.

4 Peters CA, Skoog SJ, Arant BS, et al. Summary of the AUA guideline on management of primary vesicoureteral reflux in children. $J$ Urol 2010;184:1134-44.
5 Elder JS, Diaz M. Vesicoureteral reflux--the role of bladder and bowel dysfunction. Nat Rev Urol 2013;10:640-8.

6 Hunziker M, Colhoun E, Puri P. Prevalence and predictors of renal functional abnormalities of high grade vesicoureteral reflux. J Urol 2013;190:1490-4.

7 Nakai $\mathrm{H}$, Kakizaki $\mathrm{H}$, Konda R, et al. Clinical characteristics of primary vesicoureteral reflux in infants: multicenter retrospective study in Japan. J Urol 2003;169:309-12.

8 Marra G, Oppezzo C, Ardissino G, et al. Severe vesicoureteral reflux and chronic renal failure: a condition peculiar to male gender? data from the ItalKid project. J Pediatr 2004;144:677-81.

9 Yeung CK, Godley ML, Dhillon HK, et al. The characteristics of primary vesico-ureteric reflux in male and female infants with prenatal hydronephrosis. Br J Urol 1997;80:319-27.

10 Capozza N, Gulia C, Heidari Bateni Z, et al. Vesicoureteral reflux in infants: what do we know about the gender prevalence by age? Eur Rev Med Pharmacol Sci 2017;21:5321-9.

11 Sillén U. Vesicoureteral reflux in infants. Pediatr Nephrol 1999;13:355-61.

12 Wennerström M, Hansson S, Jodal U, et al. Primary and acquired renal scarring in boys and girls with urinary tract infection. $J$ Pediatr 2000;136:30-4.

13 Senel E, Akbiyik F, Atayurt $\mathrm{H}$, et al. Urological problems or fecal continence during long-term follow-up of patients with anorectal malformation. Pediatr Surg Int 2010;26:683-9.

14 Stenström P, Kockum CC, Emblem R, et al. Bowel symptoms in children with anorectal malformation - a follow-up with a gender and age perspective. J Pediatr Surg 2014;49:1122-30.

15 Crabbe DC, Thomas DF, Gordon AC, et al. Use of 99mtechnetiumdimercaptosuccinic acid to study patterns of renal damage associated with prenatally detected vesicoureteral reflux. $J$ Urol 1992;148:1229-31. 